\title{
Erratum to: Changes in Coastal Benthic Algae Succession Trajectories and Assemblages Under Contrasting Nutrient and Grazer Loads
}

\author{
A. Fricke ${ }^{1,2}$ - G. A. Kopprio ${ }^{3}$ - D. Alemany ${ }^{4}$ - M. Gastaldi ${ }^{5}$ - M. Narvarte $^{5}$. \\ E. R. Parodi ${ }^{3,6} \cdot$ R. J. Lara ${ }^{3}$ - F. Hidalgo ${ }^{4}$ - A. Martínez ${ }^{7}$ E. A. Sar ${ }^{8}$ - O. Iribarne $^{4}$. \\ P. Martinetto ${ }^{4}$
}

Published online: 24 June 2015

(C) Coastal and Estuarine Research Federation 2015

\section{Erratum to: Estuaries and Coasts}

DOI 10.1007/s12237-015-9999-2

In the initial online publication, R. J. Lara's given name was incorrect. His name has been corrected in the publication as shown in this erratum.

The online version of the original article can be found at http://dx.doi.org/ 10.1007/s12237-015-9999-2.

\section{A. Fricke \\ Anna.Fricke@unice.fr}

1 Department of Marine Botany, University of Bremen, Leobener Str. NW2, 28359 Bremen, Germany

2 EA4228 ECOMERS, Faculté des Sciences, Université de Nice-Sophia Antipolis (UNS), Parc Valrose, 06108 Nice Cedex 2 , France

3 Consejo Nacional de Investigaciones Científicas y Técnicas (CONICET), Instituto Argentino de Oceanografía (IADO), Florida 4750, Bahía Blanca B8000FWB, Argentina

4 Laboratorio de Ecología, Instituto de Investigaciones Marinas y Costeras (IIMyC), CONICET - Universidad Nacional de Mar del Plata, Mar del Plata 7600, Argentina
5 Instituto de Biología Marina y Pesquera Almirante Storni (IBMPAS), Escuela Superior de Ciencias Marinas, Universidad Nacional del Comahue, Güemes 1030, 8520 San Antonio Oeste, Río Negro, Argentina

6 Departamento de Biología, Bioquímica y Farmacia, Universidad Nacional del Sur (UNS), San Juan 670, Bahía Blanca B8000FWB, Argentina

7 Departamento de Quimica, Universidad Nacional del Sur, Av. Alem 1253, Bahía Blanca B8000FWB, Argentina

8 División Ficología, Facultad de Ciencias Naturales y Museo, Universidad Nacional de La Plata, Paseo del Bosque s/n, 1900 La Plata, Argentina 